\title{
Star cluster analyses from multi-band photometry: the key advantage of SALT's $U$-band sensitivity
}

\author{
Uta Fritze - v. Alvensleben, Polychronis Papaderos, Peter Anders, \\ Thomas Lilly ${ }^{1}$, Barbara Cunow ${ }^{2}$ and Jay Gallagher ${ }^{3}$ \\ ${ }^{1}$ Universität Göttingen, Germany, ${ }^{2}$ Unisa Pretoria, South Africa, ${ }^{3}$ University of Wisconsin, \\ Madison, US \\ email: ufritze@astro.physik.uni-goettingen.de
}

\begin{abstract}
Conventionally, CMD analyses of nearby star clusters are based on observations in 2 passbands. They are plagued by considerable degeneracies between age, metallicity, distance (and extinction) that can largely be resolved by including additional passbands with $U$ being most appropriate for young star clusters and I or a NIR band for old globular clusters. For star clusters that cannot be resolved, integrated photometry in suitably selected passbands was shown to be as accurate as spectroscopy in independently revealing ages, metallicities, internal extinction, and photometric masses and their respective $1 \sigma$ uncertainties, when analysed with a dedicated analysis tool for their Spectral Energy Distributions (=SEDs) (cf. Anders et al. 2004a, b, de Grijs et al. 2003b). For external galaxies, rich star cluster populations can thus be efficiently analysed using deep exposures in 4 suitable filters. Again, the inclusion of the $U$ band significantly reduces the uncertainties in the cluster parameters. The age and metallicity distributions of star cluster systems yield valuable information about the formation history of their parent galaxies (Fritze - v. Alvensleben 2004). Here, we present our GALEV evolutionary synthesis models for star clusters of various metallicities (Anders \& Fritze - v. Alvensleben 2003), recently extended to include the time evolution of CMDs, the dedicated SED Analysis Tool AnalySED we developed, show results on the basis of HST data, and first results from our SALT PVP project on young star clusters in starburst and interacting galaxies.
\end{abstract}

Keywords. galaxies: star clusters, starburst, evolution, formation - techniques: photometric — methods: data analysis

\section{Introduction: why star clusters?}

Studies of Star Clusters (SCs) are interesting for many reasons: not only to learn about SC formation, evolution and destruction, but also because SCs are valuable benchmarks for stellar evolution models. For instance, effects and typical parameters of stellar rotation and binarity can well be studied on homogeneous (one age, one metallicity), reasonably sized samples of stars in clusters. Pixel-by-pixel analyses of HST ACS multi-band imaging data for the Tadpole galaxy, an ongoing merger, and other systems with our GALEV evolutionary synthesis models have shown that SC formation is a major - if not the dominant - mode of Star Formation (SF) in starbursts and galaxy mergers with, e.g., $70 \%$ of the total $U$-band light coming from young SCs in the Tadpole, not only in the main body of this galaxy, but all along its 180kpc long tidal tail (de Grijs et al. 2003a).

The Antennae galaxies, a beginning merger of 2 large gas-rich spiral galaxies similar to the Milky Way and M31 feature thousands of young SCs, many of them with radii and masses in the range of Galactic GCs (Fritze - v. Alvensleben 1998, 1999, Anders \& Fritze - v. Alvensleben, submitted). The HST WFPC2 images we analysed, however, 
only cover the innermost region of this nearby system at $\sim 16 \mathrm{Mpc}$. SALT's large field of view will make a great difference here. While we can derive ages, metallicities, $E(B-V)$, masses and radii individually for all the clusters with precise enough photometry in at least 4 passbands, we cannot say which or how many of these young massive compact SCs will survive long enough to be called Globular Clusters (GCs) in the end. An older version of essentially the same phenomenon, the already elliptical-like spiral - spiral merger remnant NGC 7252, however, still features more than 150 bright and compact SCs with ages $\geqslant 600 \mathrm{Myr}$, i.e. very probably young GCs, since they have already survived the critical times during the violent relaxation process in this system. We had predicted the possibility that a secondary population of GCs could have formed in this system from our finding that - as derived from the strength of the Balmer absorption lines in the integrated spectrum of NGC 7252 - the starburst on a global scale in this merger had a tremendously high SF efficiency, $1-2$ orders of magnitude higher than in normal SF regimes and well in the range of SF efficiencies required for the formation of strongly bound, longlived clusters by hydrodynamical cluster formation models. We also had predicted the metallicity for these clusters formed from pre-enriched gas in the spirals to be around $\left(\frac{1}{2}-1\right) \cdot Z_{\odot}($ Fritze - v. Alvensleben \& Gerhard 1994a, b, Fritze - v. Alvensleben \& Burkert 1995). Soon after HST had detected the SCs, the metallicities of two brightest ones were spectroscopically confirmed to be around $\mathrm{Z}_{\odot}$ (Whitmore et al. 1993, Schweizer \& Seitzer 1993).

This shows that SCs forming in abundance in gas-rich mergers are long-lived tracers of their parent galaxy's violent (star) formation history.

Luminous elliptical galaxies are generally observed to show bimodal distributions for the optical colors (mostly $V-I$ ) of their GCs (e.g. Gebhardt \& Kissler - Patig 1999, Kundu \& Whitmore 2001), consistent with an early major merger origin of their parent galaxies but difficult to reconcile with hierarchical formation scenarios. While the blue peak is fairly universal and consistent with old and metal-poor GCs like the ones in the Milky Way halo, the red peak seems variable in position and relative height. With one color only, however, it is not possible to determine age and metallicity differences between the two populations. First optical-NIR color distributions seem to show more structure than the optical ones (Kissler - Patig et al. 2002, Puzia et al. 2002, Hempel et al. 2003).

Using GALEV Evolutionary Synthesis models for SCs of various metallicities, we showed that secondary GCs from spiral - spiral mergers can well explain the red $(V-I)$ peak of the $\mathrm{GC}$ color distribution in $\mathrm{E} / \mathrm{S} 0 \mathrm{~s}$, but also that a wide range of combinations of age and metallicity can hide within 1 optical color peak but should split up visibly in an optical-NIR color like $(V-K)$ (Fritze - v. Alvensleben 2004). GC multi-color distributions hence provide a valuable key to the formation history of their parent galaxies. SALT's extraordinary $U$-band sensitivity and its large field of view offer a great potential here, in particular with its future NIR arm.

Therefore, we have set out to study SC formation and evolution in a wide range of nearby galaxies with SALT, from normal galaxies with quiescent SF all through the strongest starbursts in massive gas-rich mergers.

\section{The role of the $U$-band}

Our Evolutionary Synthesis models GALEV for star clusters and galaxies (http://www . astro.physik.uni-goettingen.de/ galev) calculate the time evolution of CMDs, integrated spectra, luminosities, colors, emission and absorption line strengths from 4Myr all through 16Gyr. They clearly show the great importance of the $U$-band for age and metallicity determinations of young stellar populations (cf. Fig. 1). 

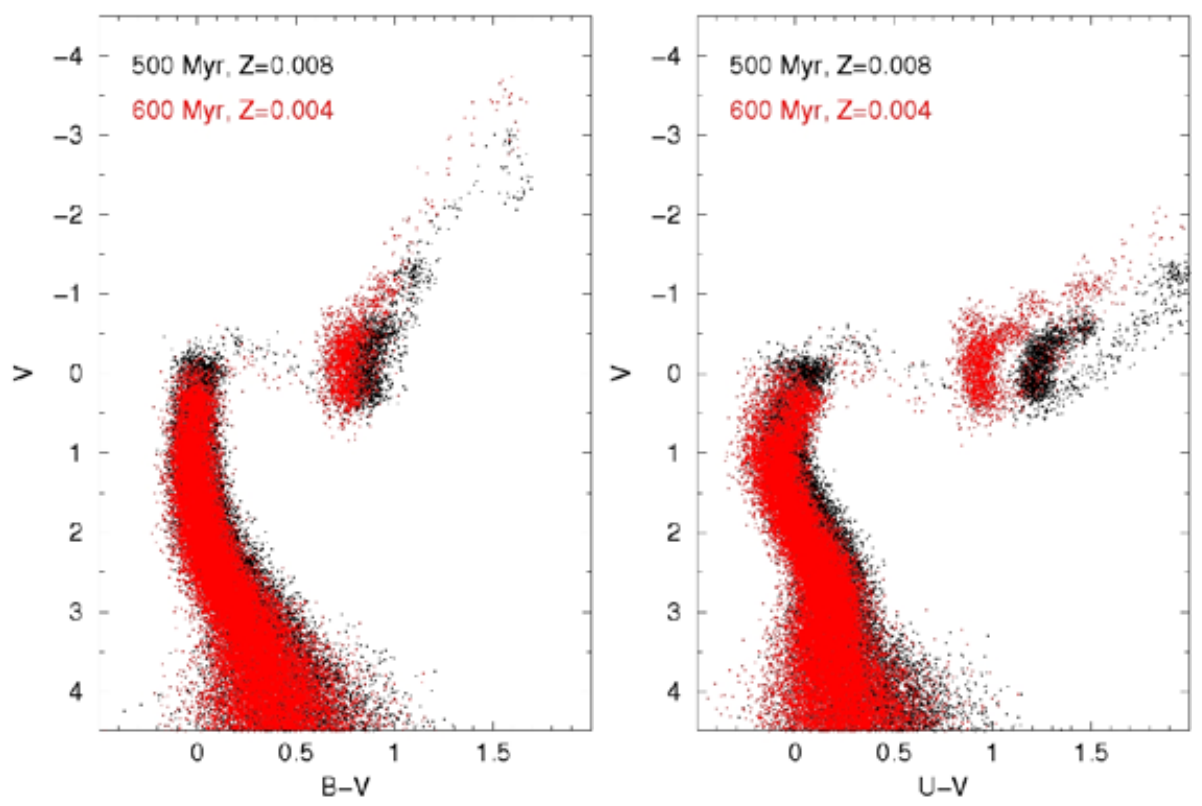

Figure 1. Model CMDs in $V-(B-V)$ and $V-(U-V)$ comparing two SCs of ages 500 and 600 Myr and metallicities $Z=0.008$ and 0.004 , respectively. While both SCs lie completely on top of each other in the $V-(B-V)$ diagram, their RGBs are very neatly separated in $V-(U-V)$.

SCs are easy to model, they contain one stellar generation with one well-defined metallicity. For a given initial mass function of the stars, e.g. Salpeter, the initial mass of the cluster determines its initial luminosity. Soon after its formation a SC starts losing mass and fades due to stellar evolution in a way that depends on metallicity. The rate of fading, however, is different in different wavelength bands. Fading in all bands is fastest in early evolutionary stages and slows down later on. In addition to this stellar evolutionary mass loss and fading, real clusters also lose stars from the tail of their Maxwellian velocity distribution due to two-body relaxation, eventually enhanced by external gravitational forces. This mass loss and fading is not included in our modelling. At a given age, the broad band luminosities in filters $U B V R I J H K$ determine the Spectral Energy Distribution (SED) of a SC. We have calculated a grid of $~ 120000$ SEDs for SCs with metallicities in the range $-1.7 \leqslant[\mathrm{Fe} / \mathrm{H}] \leqslant+0.4$, ages in the range $4 \mathrm{Myr} \ldots 14 \mathrm{Gyr}$, and extinction values $0 \leqslant E(B-V) \leqslant 1$ using Calzetti et al.'s (2000) starburst extinction law. 5 examples of SEDs for SCs of solar metallicity, $E(B-V)=0$, and ages 8, 60, 200Myr, 1 and 10Gyr are shown in Fig. 2. The mass of a cluster shifts the SEDs up and down. The strongest changes in the course of evolution are seen in the $U$-band relative to the longer wavelength bands.

Observed SC multi-band photometric SEDs can now be compared to this grid of model SEDs by means of a $\chi^{2}-$ algorithm AnalySED (Anders et al. 2004a) to obtain ages, metallicities, extinction values, and masses of all individual SCs including their respective $\pm 1 \sigma$ uncertainties. From these the age, metallicity and mass distributions of SC systems can be constructed (Anders et al. 2004b) that then give valuable clues to their parent galaxy's SF history.

Artificial star cluster analyses have identified the best optical passband combinations for SC younger (older) than a few Gyr to be $U B R I$ and $U B V I$. In any case, the $U$-band is important for ages, metallicities and extinctions of young stellar populations, while the 


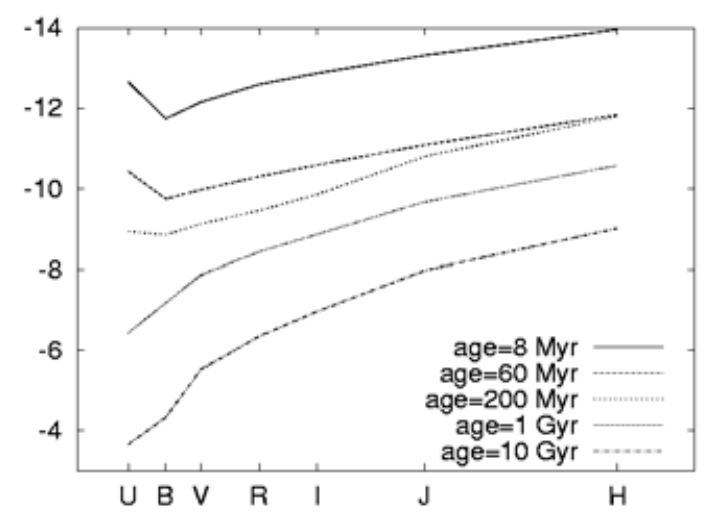

Figure 2. Model SEDs $U B V R I H$ for SCs of solar metallicity, $E(B-V)=0$, and ages 8,60 , 200Myr, 1 and 10Gyr.

NIR is important for metallicities of old stellar populations. A long wavelength basis and good photometric accuracy $(<0.1 \mathrm{mag})$ are essential in any case (Anders et al. 2004b).

We stress that at typical photometric accuracies $(\sim 0.05 \mathrm{mag})$, broad band photometry with useful passband combinations is as powerful in disentangling ages and metallicities (and extinction) as is spectroscopy with typical S/N (cf. also Cardiel et al. 2003).

\section{SALTICAM PVP observations of NGC 1487}

NGC 1487 is an ongoing merger of two spiral galaxies at a distance of $11.8 \mathrm{Mpc}$ with two pronounced tidal tails. We are interested in its SC population as described above (ages, masses, metallicities, extinction), but also in closely examining the merger-induced starburst over the entire system to assess the amount of SF that goes into cluster vs. field star formation. Ten minute $U$ - and $B$-band exposures were taken with SALTICAM very early in the Performance Verification Phase (= PVP) with a point spread function (PSF) of 1.96 arcsec FWHM and are shown in Fig. 3. An overlay of $B$-band surface brightness contours on the $(U-B)$ color map clearly reveals that the blue color peaks coincide with the highest surface brightness regions. A flux-conserving unsharp masking technique, developed by Papaderos (1998) applied to the $U$-and $B$-band images reveals more than 100 compact sources fainter than 25 mag in B, a very encouraging result at this early stage and with only part of the total observing time we requested. An improvement in the PSF by about a factor 2 is expected by the time SALT/SALTICAM starts regular operations. Comparison with HST WFPC2 BVI data on the PC chip shows that with unsharp masking SALT clearly resolves a wealth of star forming complexes.

The full analysis in the way described above requires additional observations in $V$ and $I$ and longer exposure times in $U$ and $B$ to reach fainter limits. Once the photometry is performed, which for this PVP object can be tied to the available HST photometry for absolute calibration, our SED analysis tool AnalySED will immediately return ages, metallicities, extinction, and masses for all the individual SCs and star-forming complexes. 

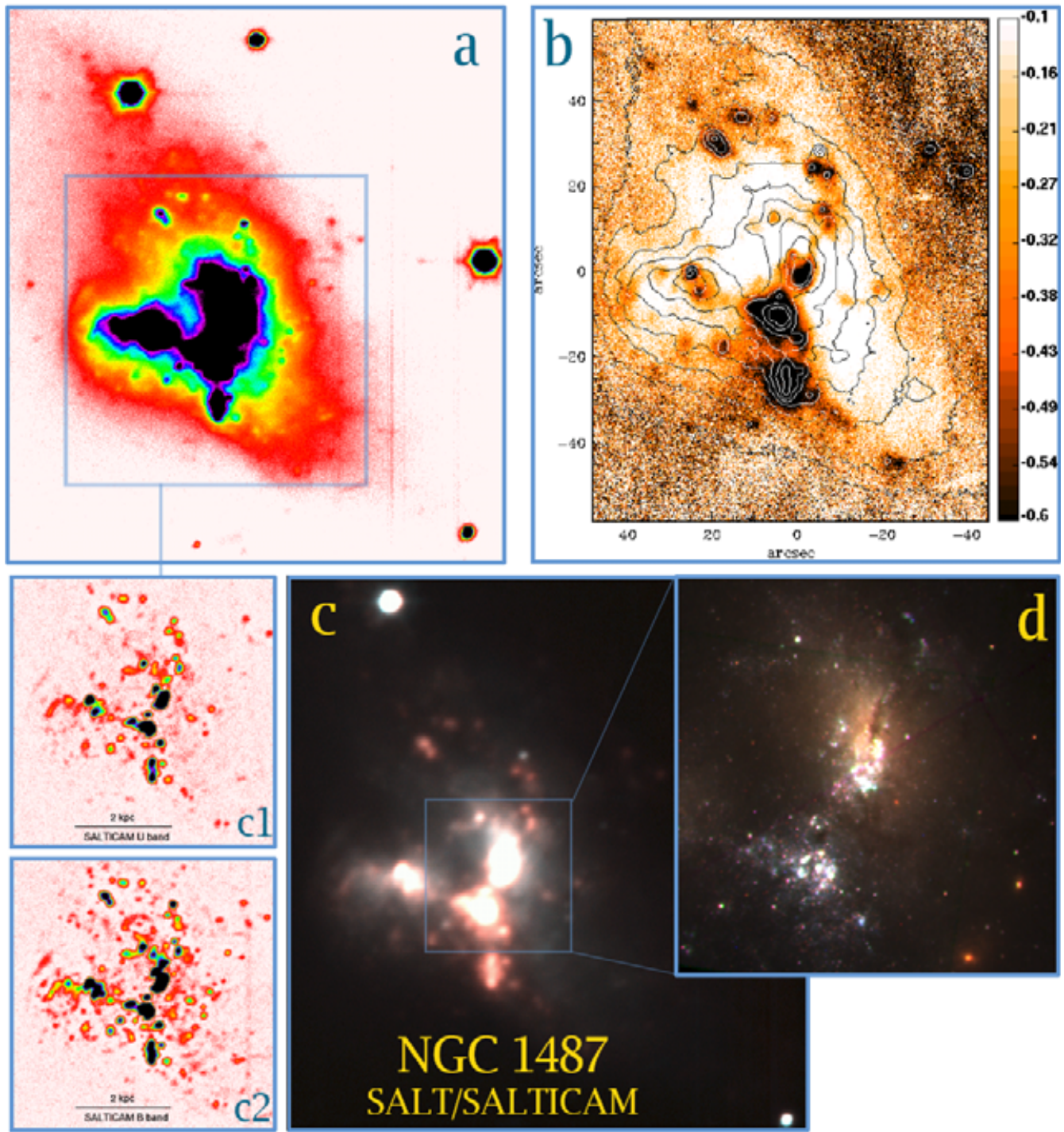

Figure 3. (a) SALTICAM B-band image of the inner region of NGC 1487, (b) B-band surface brightness contours between 20 and 25 mag $\operatorname{arcsec}^{-2}$ in steps of 0.5 overlaid with a $(U-B)$ color map, (c) true color image of the central part of NGC 1487, produced by combining $U$ and $B$ data and unsharp-masked versions of the respective broad band images (c1\&c2), (d) morphology of the central star forming component of NGC 1487 as revealed by the combination of HST WFPC2 F450W, F606W, F814W archival images.

\section{Conclusions and outlook}

SALT's large field of view and its unique $U$-band sensitivity make SALTICAM an ideal instrument for multi-band photometric analyses of young and old stellar systems, not only star clusters as shown here, but also for galaxies (see Fritze - v. Alvensleben et al., this volume). Accurate multi-band photometry allows to derive star cluster ages, metallicities, extinctions and masses including their respective $\pm 1 \sigma$ uncertainties (as well as galaxy types, redshifts, star formation histories, masses and metallicities) with accuracies comparable to those achieved in spectroscopic studies, but reaching out to much 
larger distances, and provide a valuable key to empirically constrain galaxy formation histories.

SALTICAM has demonstrated its high performance already at this early stage and provided its first and unique data set for galaxy evolution studies.

\section{Acknowledgements}

We gratefully acknowledge the SALT astronomers and SALTICAM instrument team for their very efficient and successful work and for their help with our initial data reduction problems. We also gratefully acknowledge travel support from the DFG under Fr 916/15-1 (UFvA) and PA 1228/4-1 (PP), without which we could not have attended this conference.

UFvA cordially thanks Jay Gallagher for providing her the invitation to the inauguration of SALT.

\section{References}

Anders, P. \& Fritze - v. Alvensleben, U. 2003, A\& A 401, 1063

Anders, P., Bissantz, N., Fritze - v. Alvensleben, U., de Grijs, R. 2004a, MN 347, 196

Anders, P., de Grijs, R., Fritze - v. Alvensleben, U., Bissantz, N. 2004b, MN 347, 17

Calzetti, D., Armus, L., Bohlin, R. C., et al. 2000, ApJ 533, 682

Cardiel, N., Gorgas, J., Sanchez-Blazquez, P., et al. 2003, A\&A 409, 511

Fritze - v. Alvensleben, U. 1998, A\&\&A 336, 83

Fritze - v. Alvensleben, U. 1999, A\& A 342, L25

Fritze - v. Alvensleben, U. 2004, A\&SA 414, 515

Fritze - v. Alvensleben, U. \& Gerhard, O. E. 1994a, A\&A 285, 751

Fritze - v. Alvensleben, U. \& Gerhard, O. E. 1994b, A\&A 285, 775

Fritze - v. Alvensleben, U. \& Burkert, A. 1995, A\&A 300, 58

Gebhardt, K. \& Kissler - Patig, M. 1999, AJ 118, 1526

de Grijs, R., Lee, J., Mora Herrera, C., Fritze - v. Alvensleben, U., Anders, P. 2003a, New Astron. 8, 155

de Grijs, R., Fritze - v. Alvensleben, U., Anders, P., et al. 2003b, MN 342, 259

Hempel, M., Hilker, M., Kissler - Patig, M., et al. 2003, AछA 405, 487

Kissler - Patig, M., Brodie, J. P., Minniti, D. 2002, A\&\&A 391, 441

Kundu, A. \& Whitmore, B. C. 2001, AJ 121, 2950

Papaderos, P. 1998, PhD Thesis, Universität Göttingen

Puzia, T. H., Zepf, S. E., Kissler - Patig, M., et al. 2002, A\&SA 395, 45

Schweizer, F. \& Seitzer, P. 1993, ApJ 417, L29

Whitmore, B. C., Schweizer, F., Leitherer, C., et al. 1993, AJ 106, 1354

\section{Discussion}

Gouliermis: (1)Do you have a feeling about the size of these clusters? (2) Is your $\chi^{2}$ analysis taking into account a mass function? - A Salpeter?

FRITZE: (1)From HST observations we know that individual star clusters have halflight radii of order $4 \mathrm{pc}$, they typically cluster into strong complexes of larger size. For starburst galaxies nearby, SALT can clearly resolve them under good seeing, in particular if supported by appropriate techniques like unsharp masking as I showed on the images obtained. (2) The star cluster models in the grid to which the SED analysis tool compares an observed SED have to assume a stellar IMF and we use Salpeter.

PUZIA: How much better would one break the age-metallicity degeneracy with mid-IR colours? 
FRITZE: For star clusters younger than a few Gyr, optical, i.e. $U B(V$ or $R) I$ allow for decent separations, older clusters take significant advantage of an additional VNIR passband. The mid-IR is not included in our models at the present stage due to uncertainties/controversies on appropriate stellar templates in this wavelength region (PAH etc.). 